\title{
High Resolution Imaging at mm-Wavelengths with the Hat Creek Array
}

\author{
W.C. Erickson, L.G. Mundy, and A. Grossman \\ Astronomy Department, Univ. of Maryland \\ College Park, MD 20742, USA
}

The Hat Creek mm-wavelength array, operated by the Berkeley-Illinois-Maryland Association (BIMA), is undergoing an expansion to nine telescopes. The first six of these telescopes will be operational in January, 1993. The telescopes are relatively small, $6 \mathrm{~m}$ in diameter, and are moved on rubber-tired dollies so it will be possible to transport them on roads in the vicinity of the main array. Initially, we plan to place two of the telescopes at outrigger sites located 0.5 and $1.0 \mathrm{~km}$ from the main array. Use of these outrigger telescopes will yield angular resolutions of $0.45 \mathrm{arc}-\mathrm{sec}$ at $2.7 \mathrm{~mm}$ wavelength and 0.21 arc-sec at $1.2 \mathrm{~mm}$ wavelength. When all nine telescopes are operational, reasonable image quality will be obtained with a single 12-hour observation. The maximum sidelobe amplitude depends upon source declination, but is about $16 \%$. Figures 1,2 , and 3 show the uv coverage and beamshapes which will be achieved at various declinations.

We plan to use self-calibration techniques to correct for atmospheric phase variations. In addition, it has been found that, under normal conditions, atmospheric emission in the direction of observation is well correlated with atmospheric phase delays. The idea is that the atmospheric emission and the phase variations are both caused by the water vapor column density and temperature along the paths through the atmosphere to each antenna in the interferometer. Hardware has been constructed to test this concept and we plan to actively pursue the possibility of using measurements of variations in atmospheric emission to estimate the phase fluctuations.

Communication with the outrigger telescopes will be by means of a high-quality, analog, optical link. We have tested the operation of this link on the existing telescopes. Two different fibers were tested, a normal single-mode fiber and a special, phase-stable fiber produced by Sumitomo. Both fibers operated successfully 
and yielded stable radio source fringes. In both cases the phase variations fell well within the range of the cable length measurement and compensation system, and the phase variations accurately tracked the outside air temperature. The thermal coefficient of delay for the regular single-mode fiber was estimated to be $6.0 \mathrm{ppm} /{ }^{\circ} \mathrm{C}$ and for the phase-stable fiber it was estimated to be 0.3 to $0.5 \mathrm{ppm} /{ }^{\circ} \mathrm{C}$.

When it is completed, this high resolution system will be used to map star forming regions and protoplanetary nebulae on spacial scales of 30 to $60 \mathrm{AU}$. It will also be used to make high resolution maps of active galactic nuclei and of solar flare regions.
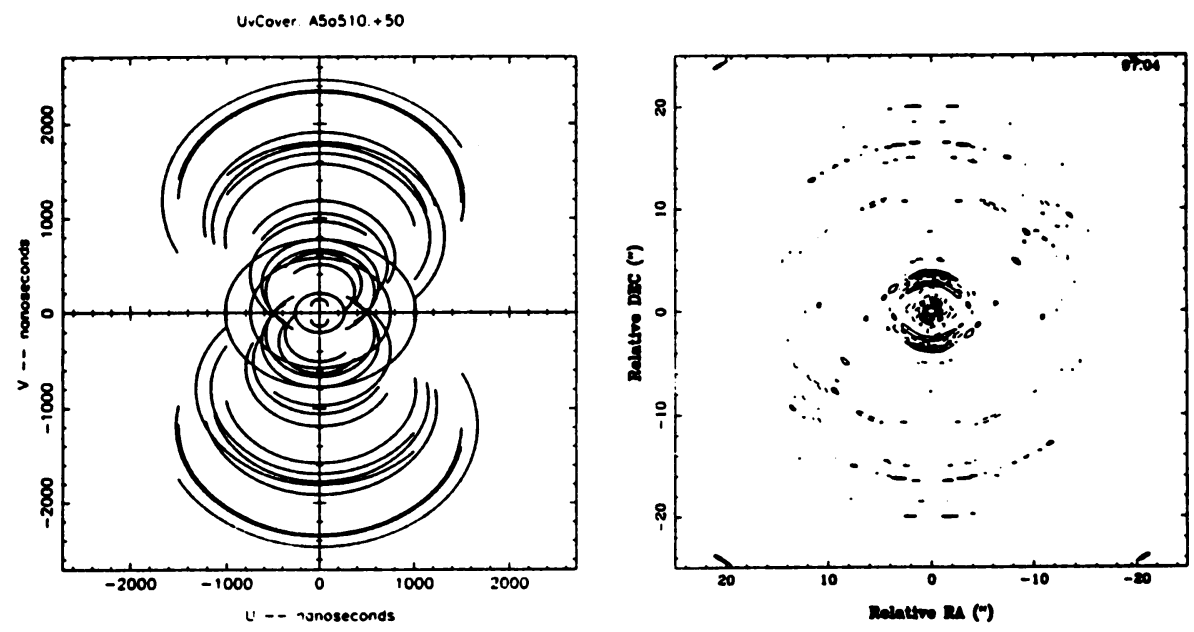

Figure 1. The uv coverage and beam pattern which will be produced by the high-resolution system at a declination of $+50^{\circ}$ with a single 12 -hour track. 

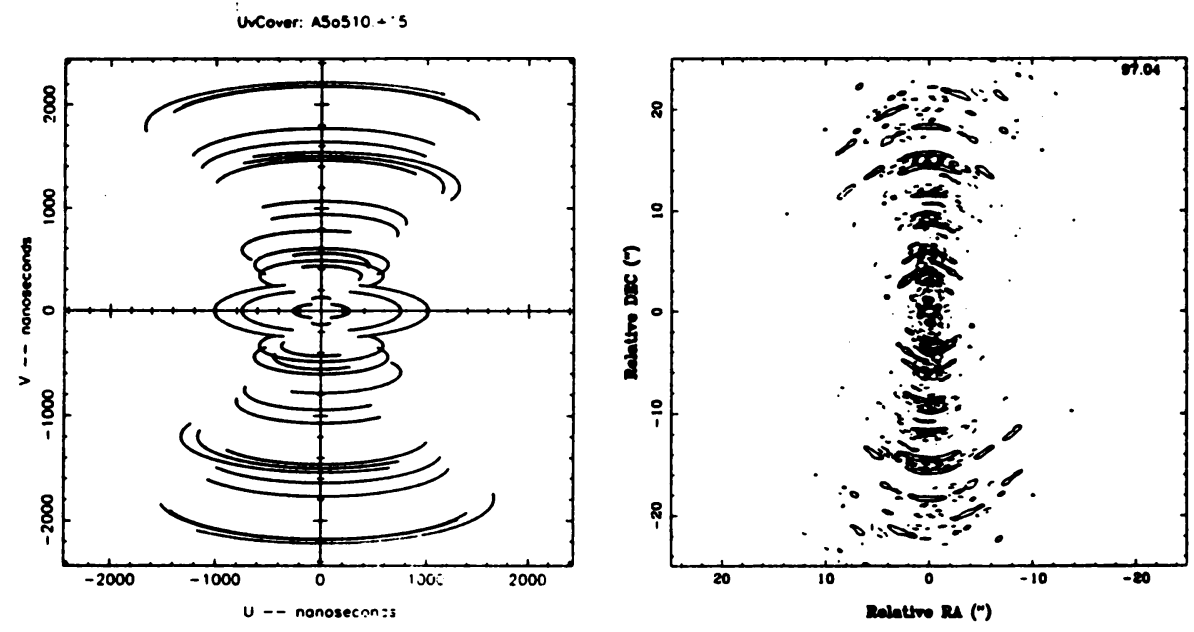

Figure 2. The uv coverage and beam pattern which will be produced by the high-resolution system at a declination of $+15^{\circ}$ with a single 12 -hour track.
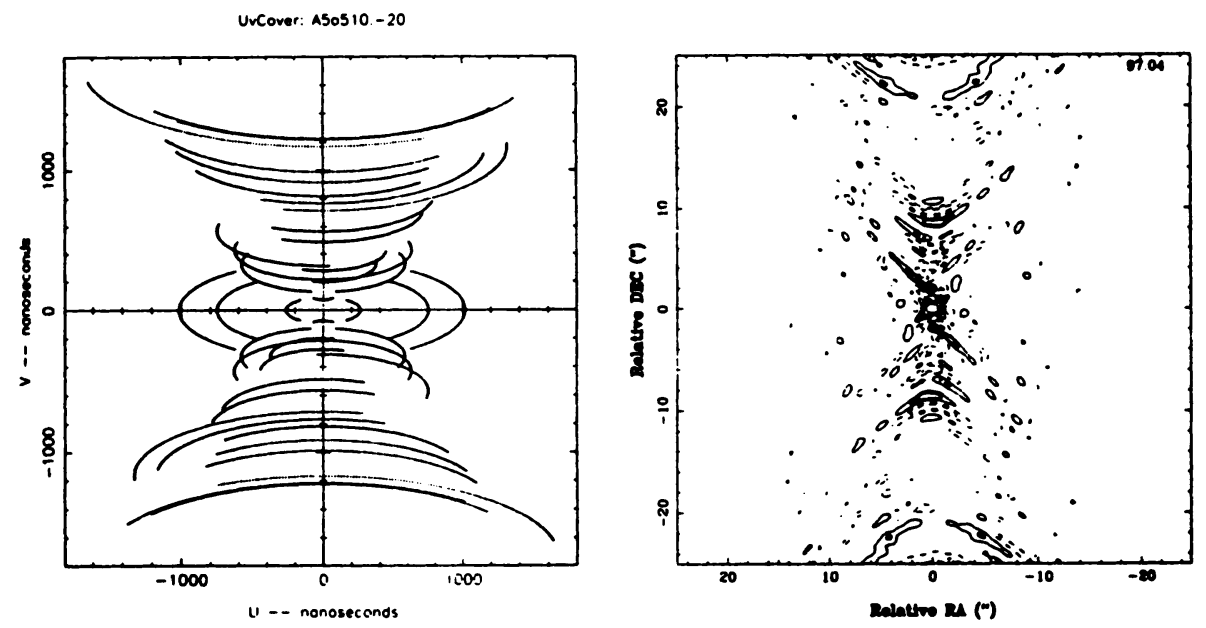

Figure 3. The uv coverage and beam pattern which will be produced by the high-resolution system at a declination of $-20^{\circ}$ with a single 12-hour track. 